\title{
A Multi-Objective, Linked-Simulation-Optimization of Henry's Saltwater Intrusion Problem using HST3D and Box's Method
}

\author{
Emily Curtis and Robert Willis \\ Department of Environmental Resources Engineering \\ Humboldt State University \\ Arcata, California 95521 USA
}

Received: 15 March 2011 Accepted: 17 September 2011

\begin{abstract}
A multi-objective linked simulation and optimization (LSO) model for a three dimensional saltwater intrusion problem has been developed. The LSO employs HST3D and Box's algorithm. The weighting method of multi-objective optimization solves the multi-objective planning problem. The model is capable of determining the optimal pumping rates that minimize the saline concentration at the well sites, as well as, the cost associated with pumping, while satisfying an exogenous water demand. The model is valid for a wide range of applications as explicitly defined by the simulation model. The application of the model was limited to a modified version of Henry's saltwater intrusion problem. Henry's problem was modified to include a third spatial dimension and well sites. The multi-objective optimization yielded explicit tradeoff information between the two objectives.
\end{abstract}

\section{INTRODUCTION}

The issue of saltwater intrusion is an important water resources problem. Increasing agricultural and domestic water demand-and the resultant rise in groundwater mining-have increased seawater intrusion in many coastal aquifers.

The objective of this study is to develop a multi-objective optimization model to address groundwater extraction in coastal aquifers. The objectives of the model are to minimize the seawater intrusion and the cost associated with pumping.

Henry's problem [1] simulates saltwater intrusion for a small two dimensional cross section. It was chosen as the prototype system for this study because a quasi-analytical solution exists, and it has been the basis for a number of saltwater intrusion studies [2-4]. Henry's original problem was modified to include a third spatial dimension with extraction sites.

The control and state variables (pumping rates and concentration) couple the multi-objective optimization model to a highly complex simulation model. Seawater intrusion is simulated by Heat and Solute Transport in Three Dimensions (HST3D) [5]. HST3D couples the nonlinear flow and solute transport equations with Box's algorithm of optimization, an methodology originally developed by Box [6] and described by Haith [7], and Willis et al. [8]. Finally, the weighting method of multiobjective optimization is used to solve the optimization. It should be noted that-to the author's knowledge-the incorporation of HST3D in an optimization routine has not been done.

\section{LITERATURE REVIEW}

The current and historical developments relating to simulation and optimization of saltwater intrusion provide a theoretical foundation for the current research. A comprehensive literature review-beginning with early studies of the process, and ending with the current trends in optimization planning and management of saltwater intrusion-follows. 


\section{a. Sharp Interface}

The sharp interface assumption treats groundwater and seawater as immiscible fluids, and allows the interface to be approximated as a surface where no diffusion takes place. When the transition zone is narrow compared with the thickness of the aquifer, the effects of mixing are ignored. The advection dispersion equation does not need to be solved.

Shamir and Dagan [9] analyzed the transient motion of saltwater intrusion and applied assumptions, such as, the sharp interface assumption, and the Dupuit approximation, to reduce the twodimensional problem to one-dimension. They compared their results to 1) an approximate analytical solution by Keulegan in 1954, which assumed a linear interface, and 2) to the Hele-Shaw experiments, which allowed for more complicated geometry at the interface. Both scenarios were reached in good agreement by their numerical approximations.

Essaid [10] developed a quasithree-dimensional finite difference model that simulated a regional, layered aquifer system, examined the dynamic fresh and saltwater interactions in Santa Cruz, California. The model was facilitated by making the sharp interface assumption. The fluid interactions between layers were coupled through the conservation of flux and pressure, and were highly nonlinear in three dimensions. By assuming horizontal flowthe Dupuit assumption-the problem was simplified. The model was verified by comparisons to the Hele-Shaw experiments by Bear and Dagan in 1964 and analytical solutions of Shamir and Dagan [9].

\section{b. Dispersive Mixing}

Pinder and Cooper [11] were among the first researchers to examine the transient motion of the saltwater front under the effects of dispersion. They used the method of characteristics and the alternating direction iterative solution procedure. For the steady state case, their model agreed with Henry's analytical solution. In the transient case, they started with two different initial conditions. Their transient solutions also approached the steady state concentrations of Henry's problem.
In the case where the diffusion process dominates the interface, and circulation induced mixing slows the encroachment of the front, a sharp interface model will over-predict the encroachment. These were the findings in Lee and Cheng's 1974 study of the Biscayne aquifer in Florida [3]. Because of the physical implications of the sharp interface assumption, they developed a steady state finite element model of the Navier-Stokes equations including dispersion. They compared their results with the analytical steady state solutions obtained by Henry, and the transient solutions achieved by Pinder and Cooper [11]. The results were in good agreement with the field data, assuming a constant dispersion coefficient.

Further development of the dispersive problem of saltwater intrusion was presented by Ségol et al. [2]. This research included the solution of three partial differential equations to remedy inconsistencies with the numerical solutions in the velocity field. The inconsistencies with velocities between elements occurred for situations where advective transport was not negligible, i.e., for large Peclet numbers. The results obtained using the Galerkin finite element method were compared to the steady state analytical Henry solution, and the transient solution by Pinder and Cooper [11].

Huyakorn et al. [12] examined the nonlinear, density dependent, coupled transient flow and solute problem in three dimensions, across a multi-layered aquifer system. Several different scenarios were presented, including one similar to the original Henry's problem with slightly different boundary conditions. The Galerkin finite element matrices were solved using slice successive relaxation with the Picard iteration scheme [12]. Results were compared to Henry's steady state and transient solutions as published by Henry, Ségol et al. (see [1]).

Voss and Souza [4] revisited the Henry problem. Specifically, they investigated the regional application with narrow transition zones of dispersion. They followed the work of Ségol et al. [2] of insuring a continuous velocity field while using finite element methods. They raised question to the legitimacy of the large dispersion coefficient of the Henry problem. 
They found the Henry problem was a valid check for highly dispersed transition zones of variable density flow. However, that Henry's problem is inappropriate with respect to a narrow transition zone, where the sharp interface assumption is usually employed. They established modeling procedures to alleviate the sources of errors associated with modeling narrow transition dispersive fronts. Specifically, they recommended 1) developing a consistent velocity field, 2) checking the purely density driven flows (as in the solute version of the Elder problem), and 3) insuring a tight spatial and temporal discretization (Voss and Souza 1987).

Koch and Sharma [13] examined saltwater intrusion in the Floridian aquifer. Both United States Geological Survey (USGS) models, the Saturated Unsaturated TRAnsport model (SUTRA) and HST3D, were used to simulate the seasonal fluctuations in saltwater intrusion. A comparison indicated that the models yielded slight differences, but the differences did not affect the conclusions. The authors investigated the injection of wastewater and also the construction of a canal zone, which would maintain the water table sufficiently high during the dry season. Their results show that the latter is a viable option to impede the already encroaching brackish front [13].

\section{c. Other Approaches}

Some researchers have investigated alternatives to the classical numeric schemes. For example, the lattice Boltzmann method, (LBM) using tworelaxation-time and a directional-speed-ofsound technique, was applied to the saltwater intrusion case. Servan-Camas and Tsai [14] applied this technique to Henry's problem. The inclusion of source/sink terms, anisotropic hydraulic conductivity, and dispersive tensors were modeled. The results of their analysis were compared to an anisotropic dispersive Henry problem, as modeled by Abarca et al. [15], and were found to be in good agreement.

\section{d. Combined Simulation-Optimization}

Regardless of the algorithm chosen to perform the optimization, the optimization model must relate the control variables of the planning or design problem to the state variables describing the environmental system. The state variables, pressure, velocity, and solute concentration are described by the continuity equation, Darcy's law, and the mass-transport equation describing a single, linear decaying and/or sorbing, species.

The coupled nonlinear flow and transport equations may be related to an optimization model through an embedding approach, where the governing hydraulic equations are treated as constraints that the optimization model must satisfy. A difficulty associated with the embedding approach lies in evaluating the coupled nonlinear partial differential equations in the constraint set at each iterative objective evaluation (see [8] and Gharbi and Peralta [16]).

The response matrix (RM) technique involves an independent simulation model that generates the system's response to pumping and creates the response matrix, which is then used by the management model to evaluate the objective. The problem with the RM technique is the imposed linearity restrictions.

Finally, the linked-simulationoptimization (LSO) approach is another method of relating the control variables and the state variables of the system. In this approach there are no restrictions on either the dimensionality or the linearity of the response matrix. A highly complex simulation model may be linked to a general optimization routine. It is for these reasons the LSO approach is utilized.

\section{e. Groundwater Optimization Models}

Early theoretical development in the optimal control of saltwater intrusion is presented by Cummings [17], and Cummings and McFarland [18]. Their theoretical developments are concerned with extensive economic models that maximize net benefits of the groundwater reserves [18]. A Lagrangian function, and the Kuhn-Tucker necessary conditions were developed to identify optimal net benefits [17].

The reclamation problem was further developed by Gorelick et al. [19]. Their use of the Modular In-Core Nonlinear 
Optimization System software (MINOS) made direct incorporation of a complex simulation model with optimization possible. The SUTRA model was used as the subroutine to simulate groundwater flow and transport in the nonlinear optimization procedure [19].

Another nonlinear optimization strategy for groundwater management was developed by Willis and Finney [8] that allowed for the nonlinear and non-convex solution of groundwater optimization models. They applied finite difference approximations of the coupled Boussinesq equation with quasi-linearization. [8]. Both quasi-linearization and Lagrangian methods were used to generate the solution of the optimization.

Jones et al. [20] continued the development of nonlinear optimization procedures with the incorporation of a differential dynamic programming algorithm and MINOS. They found this approach to significantly improve the primary issue of dimensionality associated with the embedding technique.

A regional quasi-three dimensional finite difference model representing saltwater intrusion to the Jakarta, multilayered, groundwater basin, was developed by Willis et al. [8]. The simulation model was linked to two optimization routines: MINOS and Box's algorithm. Box's algorithm improved the difficulties that MINOS encountered dealing with multiple local optimums, often associated with multiobjective optimization. Solutions were improved upon by up to $20 \%$ [21].

Gharbi and Peralta [16] applied the embedding approach of weighted multiobjective optimization to saltwater intrusion and used MINOS as the solution method. The study asserted that large scale planning problems solved with embedding approach can successfully be implemented.

The embedding approach using the boundary value simulation of saltwater intrusion along with a dynamic multiobjective optimization model was developed by Reinelt [22] to investigate the saltwater intrusion problem in the Salinas Valley of California. The economic model focuses on the externality posed by saltwater intrusion of coastal aquifers used for large scale agriculture and the tradeoffs associated with the individual's decision making [22].

\section{f. Heuristic Optimization}

The following section focuses on the non-derivative class of heuristic optimization algorithms employed in this study. There are a number of optimization models of this class: the complex method (Box's algorithm), genetic algorithms, evolutionary strategies, tabu search and simulated annealing [23]. The primary advantage of these algorithms is that the derivatives of the governing equations, or constraint set when applicable, do not need to be evaluated. Another advantage is the broad applicability to general design scenarios. In addition, the general search algorithms can be more robust at locating solutions [24]. A principal disadvantage is that the global optimum cannot explicitly be calculated. Thus, reliance upon successive changes to the starting conditions to guarantee convergence, is required [7]. Another disadvantage to this approach is that often large number of calls to the simulation model are required, which extend computation time.

Rogers and Dowla [25] posed a remediation plan for a stochastic groundwater aquifer with multiple contaminant plumes and optimized an artificial neural network (ANN). The ANN first learned to predict the flow and transport output, the ANN then searched through a vector of management scenarios to determine the optimal one which satisfied the objectives. The results of the study were compared with a quasi-Newton search. The results were consistent for both methodologies. And-contrary to the above listed disadvantage - the ANN yielded less reliance on the simulation model, and therefore, less computational effort [25].

Simulated annealing optimization was applied to a large scale, multimanagement period, multi-objective groundwater remediation case by Rizzo and Dougherty [26]. The deterministic simulation model, SUTRA, was modified to be site specific [26]. The authors claim fast convergence to nearly optimal solutions.

Cheng et al. [27] applied a genetic algorithm to a saltwater intruded aquifer in search of optimal pumping rates. The simulation model employed was simplified by the sharp interface assumption, the Dupuit approximation-reducing the 
dimensionality, and neglect of storativityreducing it to steady state. The authors asserted that gradient based methods often fail under the discontinuous space where multiple local optima exist [27], which was also the case for Willis et al. [8]. Several test cases, where the objective was to maximize pumping, were employed. The authors note the importance of restarting the optimization with different initial populations in order to obtain a global solution.

\section{MODEL DEVELOPMENT}

The following section reveals the models and procedures used. The general equations, boundary conditions, and initial conditions are discussed, as well as, the linkages between the different subprograms of the optimization methodology.

\section{a. Simulation Model}

The simulation model used to predict the state variables of the groundwater system was HST3D [28]. The general equations describing groundwater flow and solute transport employed by the HST3D simulator are given in [28]:

1) the continuity equation (conservation of mass):

$$
\frac{\partial(\varepsilon \rho)}{\partial t}=\nabla \cdot \rho \frac{\mathbb{k}}{\mu}(\nabla p+\rho g)+q \rho^{*}
$$

where

$\varepsilon \quad$ is the effective porosity (no units)

$\rho$ is the fluid density $\left(\mathrm{kg} / \mathrm{m}^{3}\right)$

$t$ is the time (s)

$\mathbb{k}$ is the porous-medium permeability tensor $\left(\mathrm{m}^{2}\right)$

$\mu \quad$ is the fluid viscosity $(\mathrm{kg} / \mathrm{m} \cdot \mathrm{s})$

$p$ is the fluid pressure $(\mathrm{Pa})$

$g$ is the gravitational constant $\left(\mathrm{m} / \mathrm{s}^{2}\right)$

$q$ is the fluid-source flow rate $\left(\mathrm{m}^{3} / \mathrm{m}^{3} \cdot \mathrm{s}\right)$

$\rho^{*}$ is the density of a fluid source $\left(\mathrm{kg} / \mathrm{m}^{3}\right)$

2) Darcy's equation (conservation of momentum):

$$
\mathbb{v}=-\frac{\mathbb{k}}{\varepsilon \mu}(\nabla p+\rho g)
$$

where $\mathbb{v}$ is the interstitial-velocity vector $(\mathrm{m} / \mathrm{s})$ and

3) The advection-dispersion equation:

$$
\begin{aligned}
& \frac{\partial(\varepsilon \rho c)}{\partial t}=\nabla \cdot \varepsilon \rho\left(\mathbb{D}_{s}+\mathbb{D}_{m} \mathbb{I}\right) \nabla c \\
& \quad-\nabla \varepsilon \rho \mathbb{V} c-\lambda \varepsilon \rho c-\rho_{b} R_{f s}+q \rho^{*} c^{*}
\end{aligned}
$$

where

$c$ is the mass fraction of solute in the fluid phase (no units)

$\mathbb{D}_{s}$ is the mechanical dispersion coefficient tensor $\left(\mathrm{m}^{2} / \mathrm{s}\right)$

$\mathbb{D}_{m}$ is the effective molecular diffusivity of the solute $\left(\mathrm{m}^{2} / \mathrm{s}\right)$

II is the identity matrix of rank 3 (no units)

$\lambda$ is the linear-decay rate constant $\left(\mathrm{s}^{-1}\right)$

$\rho_{b}$ is the bulk density of the porous medium $\left(\mathrm{kg} / \mathrm{m}^{3}\right)$

$R_{f s}$ is the transfer rate of solute from fluid to solid phase ( $\mathrm{kg}$ solute/s-kg solid phase)

$c^{*}$ is the mass fraction of solute in the fluid source (no units)

The boundary conditions may be represented by specified constant or flux, river or aquifer interaction, evapotranspiration, heat conduction and a free surface. The initial conditions are specified values of the state variables at the beginning of the simulation.

HST3D solves the flow and solute transport equations iteratively. For this study, where temperature is not involved, the pressure is solved in the flow equation first, then the mass concentration is solved for from the solute equations [28].

The system of coupled, nonlinear equations is transformed via finite differences, and can be written in general matrix notation [28]:

$$
\mathbb{A} \partial \mathfrak{u}=\mathbb{b}
$$

where

A is the matrix of the coefficient vectors of the discretized equations

$\mathbb{b}$ is the vector of initial and boundary knowns, and source or sink terms

$\partial$ un is the change in state variables vector 


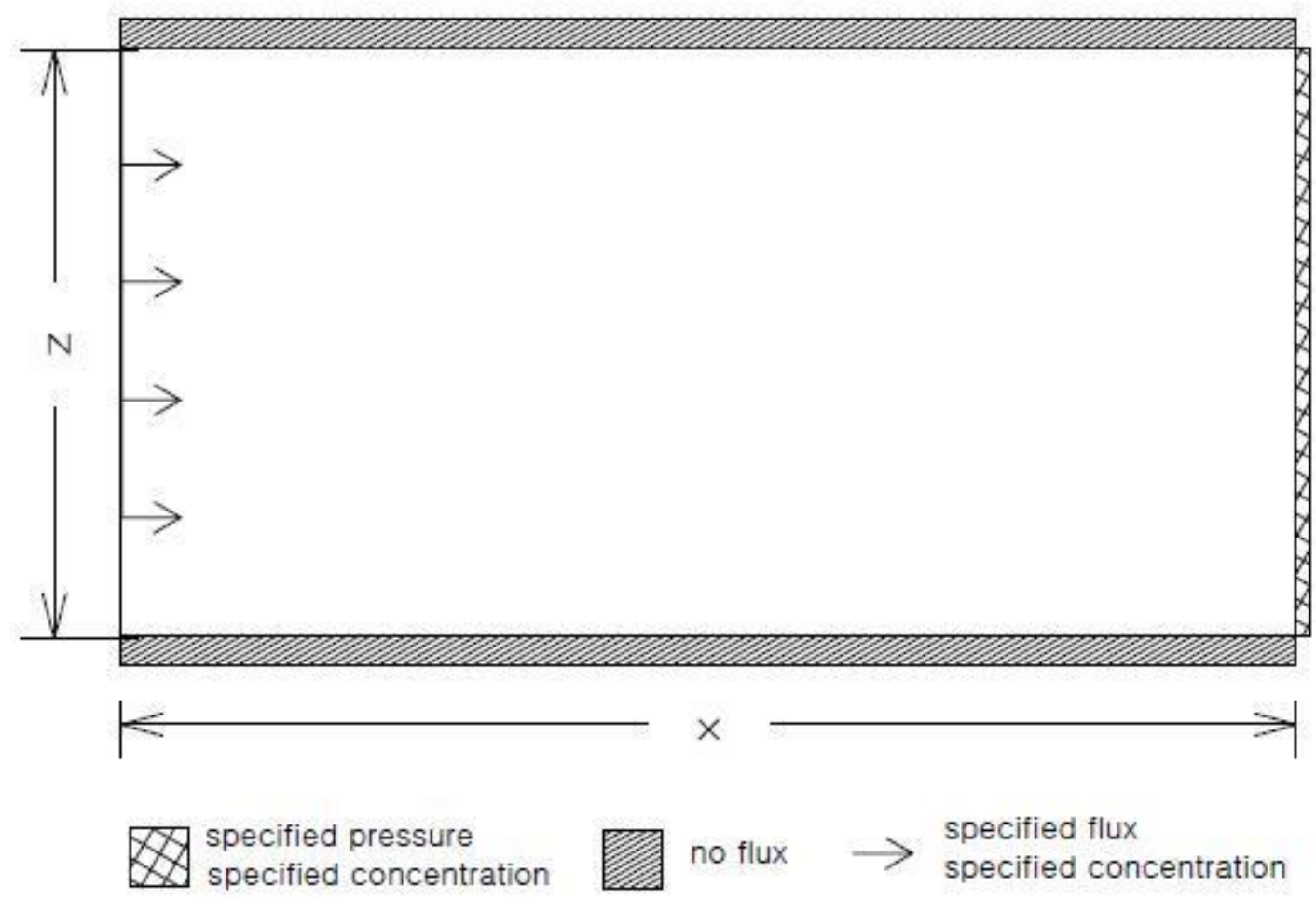

Figure 1. Schematic of Henry's Problem.

The matrix solver selected was the D4 Gaussian elimination solver developed by Price and Coats (see [28]).

\section{b. Optimization Model}

The multi-objective seawater intrusion model is expressed as a composite objective equal to the sum of two weighted objectives. The operator chooses weights that yield a tradeoff between the two objectives [29]:

subject to:

$$
\min z=\sum_{i} \omega_{i} Z_{i}(\mathbf{X})
$$

$$
\mathbf{X} \in \Omega
$$

where $\omega_{i}$ corresponds to the weight on the $i^{\text {th }}$ objective, $Z_{i}$. The objectives can represent cost, benefitsd, and water quality. The vector of decision variable $\mathbf{X}$ of any number $j$, lies in the feasible region $\Omega$.

\section{Application}

Henry's problem of saltwater intrusion was modified. The problem simulated in this study is a threedimensional transient aquifer with pumping, similar to Huyakorn et al. [12]. The mathematical representation follows that of Henry [1], Voss and Souza [4] and Ségol [2], as described by Kipp [28]. The boundary conditions that describe the system are represented in Figure 1, and mathematically as:

$$
\begin{gathered}
v_{n}(0, y, z, 0)=Q / \varepsilon x \\
p(x, y, z, 0)=\rho_{s} \mathrm{~g} z \\
v_{n}(x, y, 0, t)=0 \\
v_{n}(0, y, z, t)=0 \\
v_{n}(x, 0, z, t)=0 \\
v_{n}(x, y, z, t)=0 \\
\frac{\partial c}{\partial y}(x, 0, z, t)=0
\end{gathered}
$$




$$
\begin{aligned}
& \frac{\partial c}{\partial y}(x, y, z, t)=0 \\
& \frac{\partial c}{\partial z}(x, y, 0, t)=0 \\
& \frac{\partial c}{\partial z}(x, y, z, t)=0
\end{aligned}
$$

Where $Q$ is the freshwater influx entering the aquifer $\left(\mathrm{m}^{3} / \mathrm{s}\right)$. Table 1 summarizes additional parameters used by the simulation model.

The initial salt concentration in the aquifer is described by:

$$
\begin{aligned}
& c(0, y, z, t)=0 \\
& c(x, y, z, t)=c_{S}
\end{aligned}
$$

where $c_{s}$ is the concentration of the saltwater.

The objective of the multi objective optimization model is to minimize the cumulative salt concentration at the well sites, and the total cost of pumping at each active well site. The objective function is rescaled by the coefficient on the numeraire, formally:

$$
\begin{aligned}
\min z & =w_{1} \sum_{i}^{n} \alpha Q_{i j}^{\beta}+w_{2} c_{j} \\
& =\frac{w_{1}}{w_{2}} \sum_{i}^{n} Q_{i j}^{\beta}+c_{j} \\
& =\widehat{w} \sum_{i}^{n} \alpha Q_{i j}^{\beta}+c_{j}
\end{aligned}
$$

subject to:

$$
\sum_{i j} Q_{i j} \geq D
$$

where $\alpha$ and $\beta$ are user-specified parameters, $Q_{i j}$ is the pumping rate of well $i$ and alternative $j, c_{j}$ is the concentration of alternative $j$, and $D$ is the demand.

\begin{tabular}{|l|l|l|}
\hline Parameter & Value & Units \\
\hline$x$ & 2 & $\mathrm{~m}$ \\
\hline$y$ & 2 & $\mathrm{~m}$ \\
\hline$z$ & 1 & $\mathrm{~m}$ \\
\hline$v_{n}$ & $6.6 \times 10^{-5}$ & $1 / \mathrm{t}$ \\
\hline$\epsilon$ & 0.35 & \\
\hline$\rho_{S}$ & 1024 & $\mathrm{~kg} / \mathrm{m}^{3}$ \\
\hline$\rho_{f}$ & 1000 & $\mathrm{~kg} / \mathrm{m}^{3}$ \\
\hline$k$ & $1.020 \times 10^{-9}$ & $\mathrm{~m}^{2}$ \\
\hline$D_{m}$ & $18.8571 \times 10^{-6}$ & $\mathrm{~m}^{2} / \mathrm{s}$ \\
\hline$\left.P\right|_{Z=0}$ & 10,052 & $\mathrm{~Pa}$ \\
\hline$\Delta X, Y, Z$ & 0.1 & $\mathrm{~m}$ \\
\hline $\begin{array}{l}\text { Number of } \\
\text { nodes }\end{array}$ & 4851 & \\
\hline $\begin{array}{l}\text { Number of } \\
\text { wells }\end{array}$ & 19 & \\
\hline
\end{tabular}

Table 1. Model Parameters.

\section{d. Linked-Simulation-Optimization}

The multi-objective optimization model was solved using the LSO methodology. The LSO method links the simulation model with the optimization model. HST3D was coupled to Box's algorithm, and the multi-objective optimization routine was solved using the weighting method. The LSO begins when the optimization algorithm generates an initial feasible solution. The solution is checked to insure it satisfies the constraints. In addition to the demand constraint, there were internal constraints imposed by Box's algorithm. The internal constraints are restrictions on each of the decision variables upper and lower bounds. The number of alternatives, $P$, must satisfy $P \geq n+1$, where $n$ is the number of decision variables. The initial pumping rates are sent to the simulator. The simulator uses the pumping rates to generate the cumulative salt concentration at the well sites. The concentrations are then sent back to the optimization routine. The optimization routine then evaluates the objective, and removes the largest one. The remaining randomly generated alternatives are sequentially evaluated for their ability to satisfy the constraints. If the randomly selected alternatives don't meet the internal constraints, they are moved closer to the centroid of the feasible solutions. The worst 


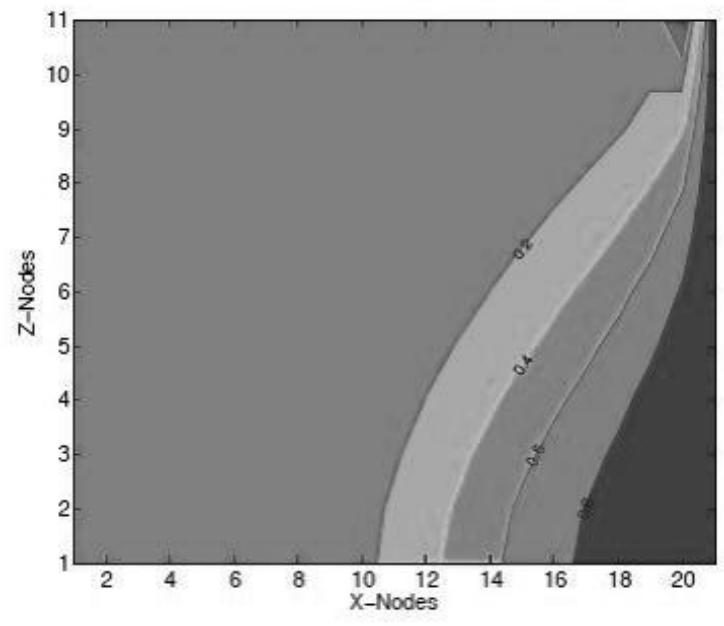

(a)

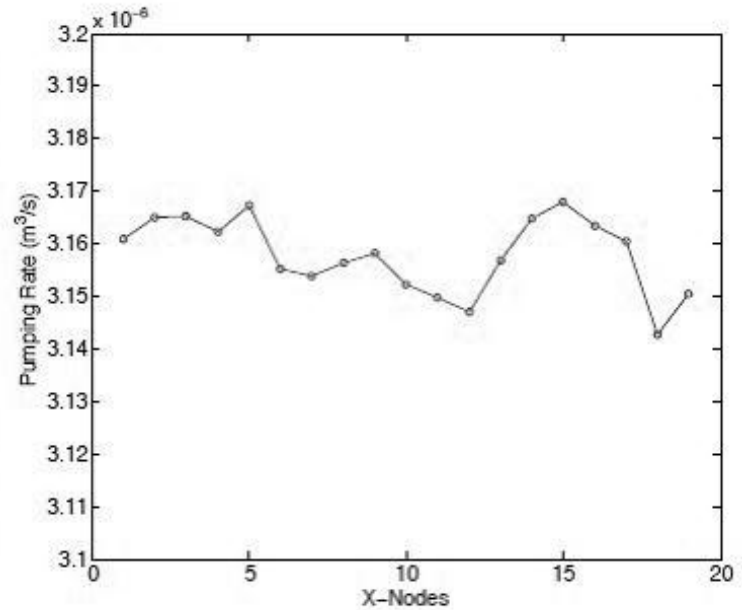

(b)

Figure 2. Cost objective dominates. a) Concentration contours, depth $y=0.5 \mathrm{~m}$; b) Pumping rates at optimal $Z_{1}$. Note: see the cover for a color version of this figure.

alternative is systematically removed each iteration. The process repeats itself until a locally optimal solution is reached, that is, until the exit criteria has been reached. For a detailed description, the reader is referred to Box [6] and Haith [7].

\section{RESULTS}

The optimization model was verified in the initial stages of development. First the simulator was verified, later the single objective case, and finally the multi-objective optimization was verified. A brief discussion of this process follows.

\section{a. Verification}

The verification of HST3D involved replicating the original Henry's problem. The domain was then expanded to include a depth, and well sites were introduced. The transient simulation approached the steady state solution.

The optimization model was verified by first analyzing a single objective. The optimal vector of pumping rates resulting from the minimization of concentration were input to HST3D. The concentrations that resulted from the simulation and optimization pumping rates were compared. The percent difference between the two concentrations was calculated for a variety of simulations with different input parameters, and resulted in a mean value of $0.02 \%$. Similarly the process was followed for the minimization of the cost objective. The weight on the concentration was set to zero, and the optimal pumping rates were again simulated. The concentrations were replicated again with the same mean percentage difference.

\section{b. Optimal Solutions}

The results of the multi-objective optimization are presented. Figure 2 a shows the concentration profile as the cost objective dominates the solution. The contours are taken at a depth of $y=0.5 \mathrm{~m}$. The figure shows the salt concentration increase from the freshwater to saltwater boundary, where the bulk of the intrusion lies along the saltwater boundary. The pumping rates that are associated with the concentration contours are shown in Figure $2 \mathrm{~b}$. They vary slightly around a mean of 3.16 $\times 10^{-6} \mathrm{~m}^{3} / \mathrm{s}$.

The concentration optimization is summarized in Figures $3 \mathrm{a}$ and $3 \mathrm{~b}$. The optimization established the pumping rates that satisfied the demand, and resulted in the smallest concentration objective. The concentration contours are taken at the 


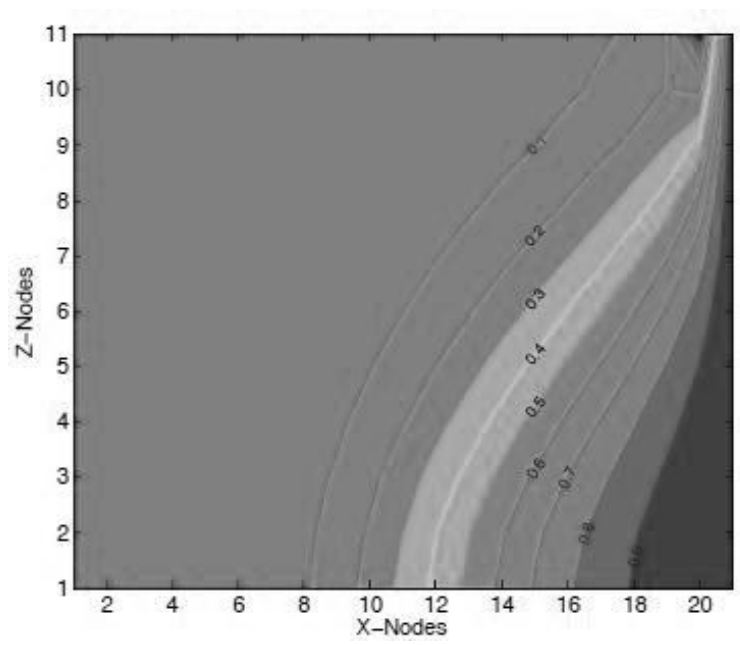

(a)

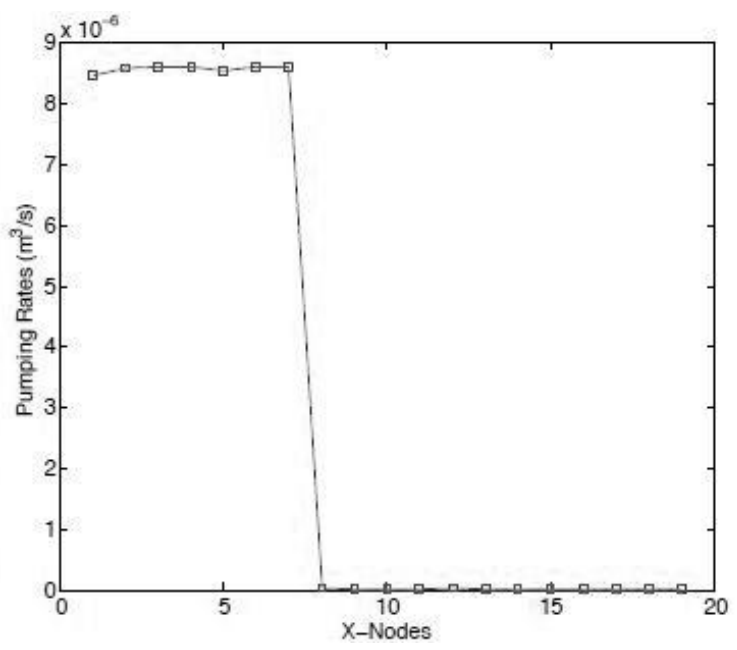

(b)

Figure 3. Concentration objective dominates. a) Concentration contours, depth $y=0.5 \mathrm{~m}$; b) Pumping rates at optimal $Z_{1}$. Note: see the cover for a color version of this figure.

same depth of $y=0.5 \mathrm{~m}$. In this case, the saltwater extends through the midpoint of the aquifer, but doesn't span the entire domain. The optimal pumping rates satisfy the demand using the 7 wells closest to the freshwater boundary, while the remaining wells were made inactive by the LSO.

As the objective weights were varied, a tradeoff analysis of the multiobjective optimization was generated (Figure 4). The feasible range of optimal solutions are bounded by the upper and lower limits of the optimization, (the single objective optimization of concentration and cost). The efficiency frontier is a convex function. The slope of each piecewise segment of the efficiency frontier represents the tradeoff between the cost and concentration objectives $(\$ / \mathrm{kg})$. Each weight, applied in the optimization yields a region with a different tradeoff value. Table 2 lists 1) the weight applied in each optimization, 2) the respective values of the objectives, 3) the tradeoff associated with each weight, 4) the execution time for a 3.33 $\mathrm{GHz}$ Intel Dual Core processor, and 4) the number of calls to the simulation model for each application.

\begin{tabular}{|c|c|c|c|c|c|}
\hline $\mathbf{W}\left(\mathbf{\times 1 0 ^ { \mathbf { 9 } }}\right)$ & $\mathbf{Z}_{\mathbf{2}} \mathbf{( k g )}$ & $\mathbf{Z}_{\mathbf{1}}\left(\mathbf{\$ \times \mathbf { 1 0 } ^ { - \mathbf { 1 0 } } )}\right.$ & slope $\mathbf{( \$ \mathbf { k g } )}$ & $\mathbf{T}(\mathbf{h})$ & calls (\#) \\
\hline 0 & 6.12 & 4.89 & 0.79 & 20.0 & 2,587 \\
\hline 25 & 7.24 & 4.07 & 0.56 & 14.7 & 1,926 \\
\hline 50 & 10.76 & 3.05 & 0.28 & 16.2 & 2,193 \\
\hline 100 & 14.7 & 2.45 & 0.16 & 12.9 & 2,812 \\
\hline 150 & 18.43 & 2.18 & 0.12 & 12.2 & 1,794 \\
\hline 250 & 22.75 & 1.96 & 0.09 & 14.3 & 2,056 \\
\hline 750 & 27.78 & 1.82 & 0.07 & 16.8 & 2,418 \\
\hline $1,000,00$ & 30.09 & 1.80 & 0.06 & 28.8 & 4,251 \\
\hline
\end{tabular}

Table 2. Efficiency frontier values. 


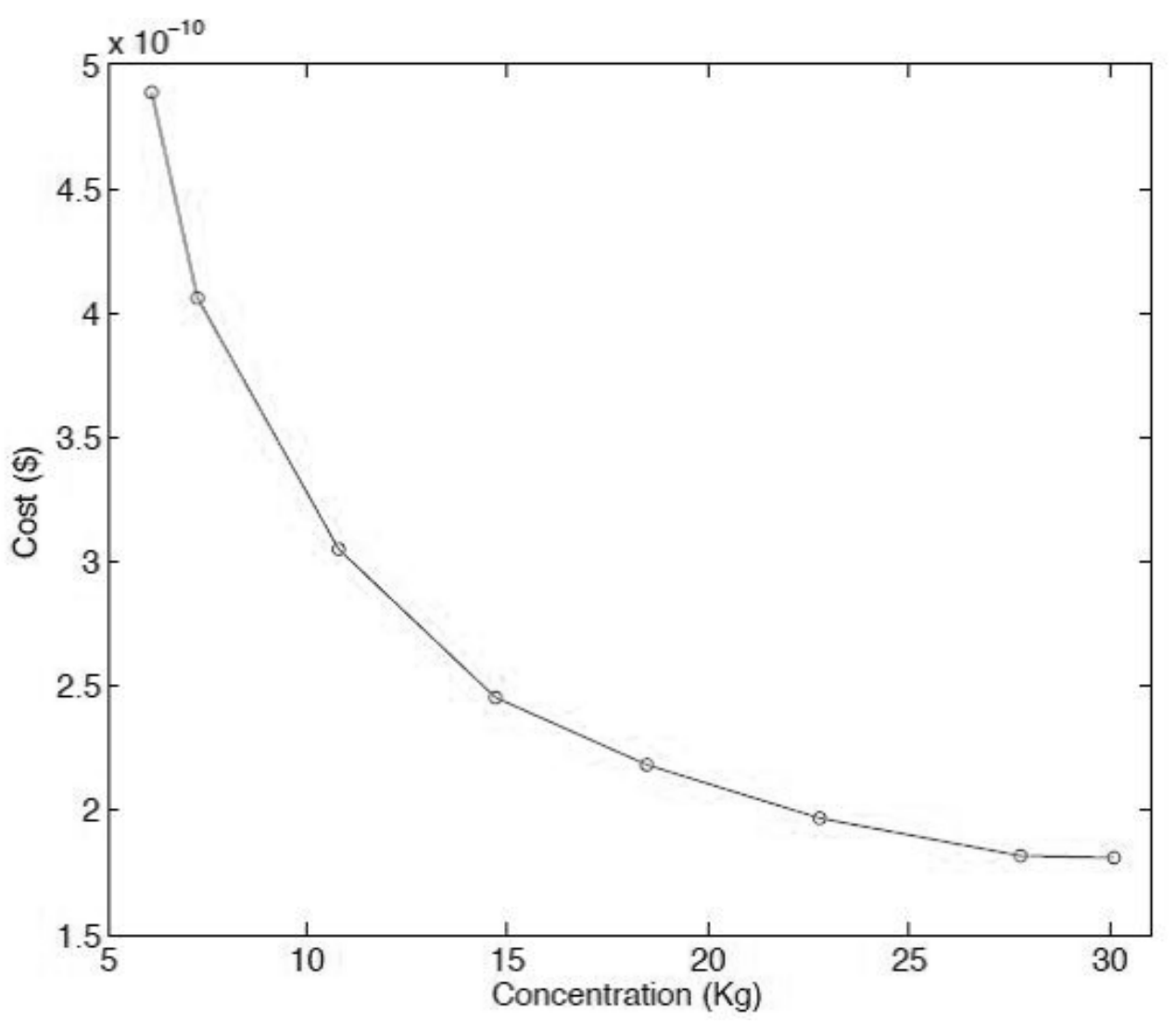

Figure 4. Efficiency frontier, $Z_{1}$ vs. $Z_{2}$ with different weights.

Specifically, the tradeoffs associated with the region of low concentrations are very steep. In this region, small increases in the concentration objective result in a large cost benefit. Conversely gradual slopes are associated with the region of higher concentrations, in this region, little cost benefit is achieved with an increase in concentration. The weights applied to the optimization that generate the region at the center of the efficiency frontier resulted in moderate slopes varying slightly between 0.12 and $0.16(\$ / \mathrm{kg})$ as seen in Table 2.

\section{CONCULSIONS AND FURTHER RESEARCH}

The objective of this study was to create a multi-objective optimization model that incorporated a complex simulation model with optimization to address the issue of groundwater planning and management with respect to seawater intrusion of coastal aquifers. The optimization model was verified and used to explore the limits of optimization within the small three dimensional prototype system.

A field scale model was developed but not pursued, because of the extensive computational resources required. Further development of regional applications are left for future research.

\section{ACKNOWLEDGEMENTS}

This research began as a collective effort between team members of a directed design course in Water Resources Engineering. Special thanks to Kevin Jensen and Michael Price who contributed to the preliminary development.

\section{REFERENCES}

1. Henry, H. R. (1964). "Interface between salt water and fresh water in a coastal aquifer". In: Cooper H.H. Jr., Kohout F.A., Henry H.R. and Glover R.E. (eds), Sea water in coastal aquifers. U.S. Geological Survey Water Supply Paper 1613-C, pp. C35-C70. 
2. Ségol, G., Pinder, G. F., and Gray, W. G. (1975). "A Galerkin-Finite Element Technique for Calculating the Transient Position of the Saltwater Front." Water Resources Research, 11(2), 343-347.

3. Lee, C.-H. and Cheng, R. T.-S. (1974). "On Seawater Encroachment in Coastal Aquifers." Water Resources Research, 10(5), 1039-1043.

4. Voss, C. I. and Souza, W. R. (1987). "Variable Density Flow and Solute Transport of Regional Aquifers Containing a Narrow FreshwaterSaltwater Transition Zone." Water Resources Research, 23(10), 18511866

5. Kipp, K. L. (1997). "Guide to the revised heat and solute transport simulator: HST3D - version 2." Water Resources Investigations Report 97-4157.

6. Box, J. M. (1965). "A new method of constrained optimization and a comparison with other methods." The Computer Journal, 8, 45-52.

7. Haith, D. A. (1982). Environmental Systems Optimization. (John Wiley and Sons, Inc., New York, N.Y).

8. Willis, R. and Finney, B. A. (1985). "Optimal Control of Nonlinear Groundwater Hydraulics: Theoretical Development and Numerical Experiments." Water Resources Research, 21(10), 1476-1482.

9. Shamir, U. and Dagan, G. (1971). "Motion of the Seawater Interface in Coastal Aquifers: A Numerical Solution." Water Resources Research, 7(3), 644657.

10. Essaid, H. I. (1990). "A Multilayered Sharp Interface Model of Coupled Freshwater and Saltwater Flow in Coastal Systems: Model Development and Application." Water Resources Research, 26(7), 1431-1454.

11. Pinder, G. F. and Cooper, H. H. (1970). "A Numerical Technique for Calculating the Transient Position of the Saltwater Front." Water Resources Research, 6(3), 875-862.

12. Huyakorn, P. S., Andersen, P. F., Mercer, J. W., and Harold O. White, J. (1987). "Saltwater Intrusion in Aquifers: Development and Testing of a ThreeDimensional Finite Element Model." Water Resources Research, 23(2), 293312.
13. Koch, M. and Sharma, A. (2003). "SUTRA and HST3D Modeling and Management of Saltwater Intrusion from Brackish Canals in Southeast Florida." University of Kassel, Germany and National Institute of Hydrology, India.

14. Servan-Camas, B. and Tsai, F. T.-C. (2010). "Two-Relaxation-Rime Lattice Boltzmann method for the anisotropic dispersive Henry problem." Water Resources Research, 46(WO2515).

15. Abarca, Elena, Jesús Carrera, Xavier Sánchez-Vila, and Marco Dentz (2007). "Anisotropic dispersive Henry problem." Advances in Water Resources 30, 913926.

16. Gharbi, A. and Peralta, R. C. (1994). "Integrated embedding optimization applied to Salt Lake valley aquifers." Water Resources Research, 30(3), 817832.

17. Cummings, R. G. (1971). "Optimum Exploitation of Groundwater Reserves with Saltwater Intrusion." Water Resources Research, 7(6), 1415-1424.

18. Cummings, R. G. and McFarland, J. W. (1974). "Groundwater Management and Salinity Control." Water Resources Research, 10(5), 909-915.

19. Gorelick, S. M. and I.Voss, C. (1984). "Aquifer Reclamation Design: The Use of Contaminant Transport Simulation CombinedWith Nonlinear Programming." Water Resources Research, 20(4), 415-427.

20. Jones, LaDon, Robert Willis, and William W.-G. Yeh (1987). "Optimal control of nonlinear groundwater hydraulics using differential dynamic programming" Water Resources Research, 23 2097-2106.

21. Finney, B. A., Samsuhadi, and WIllis, R. (1992). "Quasi-Three-Dimensional Optimization Model of Jakarta Basin." Journal of Water Resources Planning and Management, 118(1), 18-31.

22. Reinelt, P. (2005). "Seawater intrusion policy analysis with a numerical spatially heterogeneous dynamic optimization model." Water Resources Research, 41 (W05006).

23. Lee, K. Y. and El-Sharkawi, M. A. (2008). Modern Heuristic Optimization Techniques with Applications to Power Systems (John Wiley and Sons, Inc.) 
24. Andersson, J. (2001). "Multiobjective Optimization in Engineering Design," PhD thesis, Linköping University.

25. Rogers, L. L. and Dowla, F. U. (1994). "Optimization of groundwater remediation using artificial neural networks with parallel solute transport modeling." Water Resources Research, 30(2), 457-481.

26. Rizzo, D. M. and Dougherty, D. E. (1996). "Design optimization for multiple management period groundwater remediation." Water Resources Research, 32(8), 2549-2561.
27. Cheng, A. H.-D., Halhal, D., Naji, A., and Ouzaar, D. (2000). "Pumping optimization in saltwaterintruded coastal aquifers" Water Resources Research, 36(8), 2155-2165.

28. Kipp, K. L. (1987). HST3D: A Computer Code for Simulation of Heat and Solute Transport in Three-Dimensional Ground-Water Flow Systems. U.S. Geological Survey, Denver Colorado, $2^{\text {nd }}$ edition.

29. Cohon, J. and Marks, D. (1975). "A Review and Evaluation of Multiobjective Programming Techniques." Water Resources Research, 11(2), 208-220.
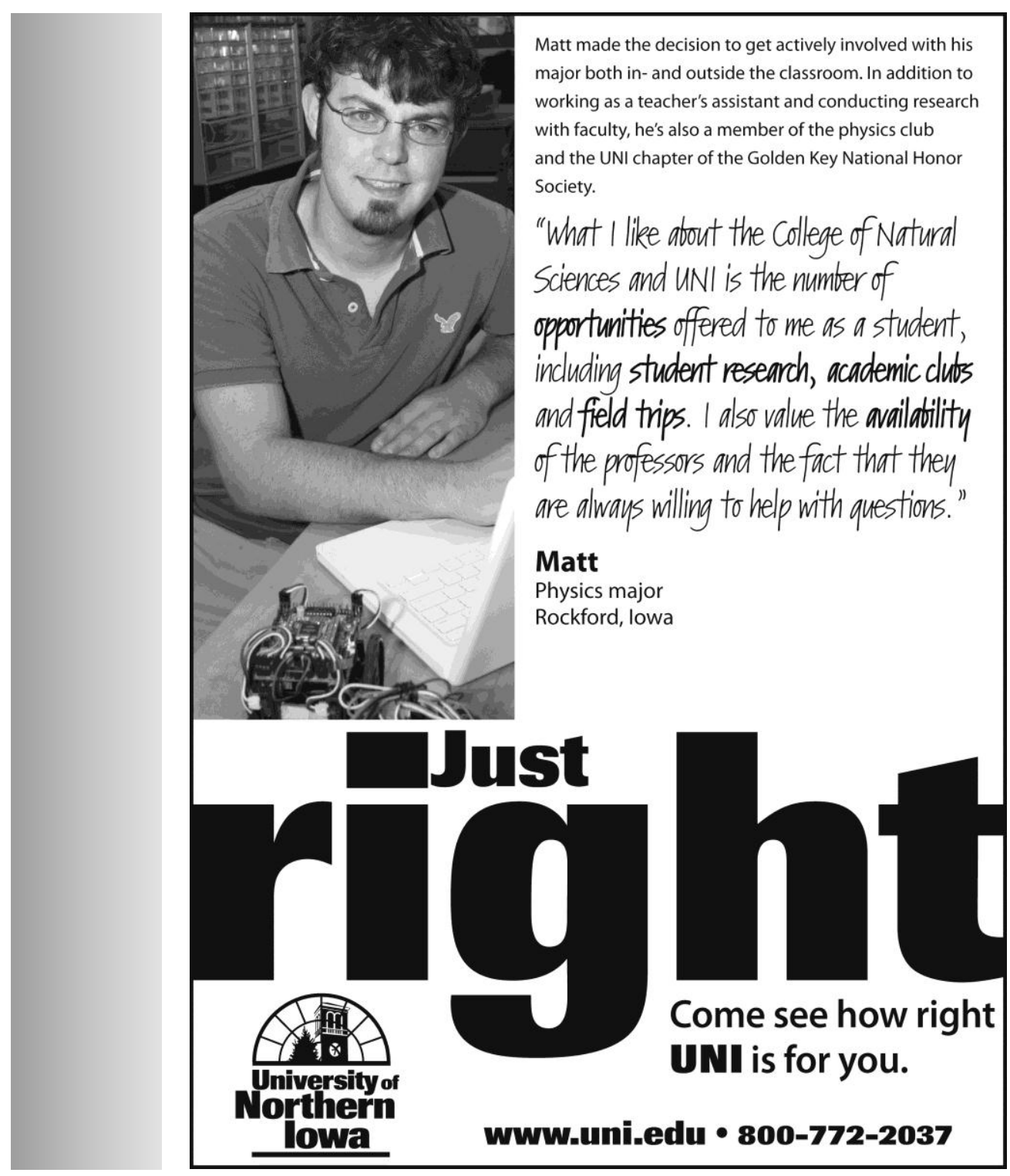